\title{
Variabilité Morphologique Et Agronomique Des Morphotypes De Voandzou (Vigna Subterranea (L.) Cultivés Dans La Zone Sahélienne Du Niger
}

\author{
Amadou Harouna Issa \\ Université Abdou Moumouni, Faculté des Sciences et Techniques, \\ Département de Biologie, Laboratoire Gestion et Valorisation de la \\ Biodiversité au Sahel, Niamey, Niger
}

Agali Alhassane

Centre Régional AGRHYMET,

Département Information et Recherche, Niamey, Niger

Sani Daouda Ousmane

Université Abdou Moumouni,

Institut de Recherche en Radio-Isotopes, Niamey, Niger

Souleymane Boureima Kodo

Université Abdou Moumouni, Faculté d'Agronomie, Niger

Doi: 10.19044/esj.2018.v14n36p377 URL:http://dx.doi.org/10.19044/esj.2018.v14n36p377

\begin{abstract}
In Niger, Bambara groundnut is mainly grown by women in pure culture on small areas and without improved technics. However, improving the production of this crop can help to ensure food security. Knowledge of Bambara groundnut's diversity will allow its use in breeding programs. This study concerns the agro-morphological characterization in the Sahelian agroclimatic zone of fourteen (14) morphotypes of Bambara groundnut identified in the Nigerien accessions collection. The data used are collected through an agronomic test using a complete random block design, with four repetitions. Twelve (12) characters including 01 phenological, 08 morphological and 03 related to the yield components at harvest were evaluated. Descriptive statistical analysis showed that the coefficients of variation ranged from $10.3 \%$ (early flowering) to $72 \%$ (seeds weight per plant). Significantly high values ( $\mathrm{CV}>20 \%$ ) were observed for 07 characters out of 12 analyzed. Agromorphological characterization revealed significant differences between the 09 parameters of the morphotypes. It was found that the $\mathrm{Ne}-10$ and $\mathrm{Ne}-14$ morphotypes are more efficient in grain yield, with 10.8 and $9.6 \mathrm{~g} /$ plant, respectively. Late-flowering morphotype (Ne-01) had the lowest yield. Strong correlations were observed in the morphological parameters, with $r=$
\end{abstract}


0.82 between the petiole length and the width of the terminal leaflet. A correlation of the same order was obtained between the wingspan of the plant and the width of the terminal leaflet, with $r=0.76$. For yield parameters, strong correlations were observed between seed weight per plant and pod weight per plant $(\mathrm{r}=0.96)$, and between same seed weight and number of pods per plant $(r=0.87)$. The dendrogram classified the morphotypes into 4 groups, with the Ne-14 and Ne-01 morphotypes each forming two isolated groups, the $\mathrm{Ne}-04$ and $\mathrm{Ne}-08$ morphotypes forming the third group and the 10 others together constituting the fourth group.

Keywords: Vigna subterranea, Sahelian zone, Diversity, Morphotypes, Niger

\section{Resume}

Au Niger, le voandzou est principalement cultivé par les femmes, en culture pure sur des petites surfaces et sans techniques améliorées, alors que l'amélioration de la production de cette culture peut contribuer à assurer la sécurité alimentaire. En effet, la maitrise de la diversité du voandzou permettra sans doute sa prise en compte dans les programmes de sélection variétale. Cette étude aborde la caractérisation agro-morphologique dans la zone agro-climatique sahélienne de quatorze (14) morphotypes de voandzou identifiés dans la collection des accessions du Niger. Les données utilisées sont collectées à travers un essai agronomique mis en place sur un dispositif en blocs aléatoires complets, avec quatre répétitions. Douze (12) caractères, dont 01 phénologique, 08 morphologiques et 03 liés aux composantes du rendement à la récolte, ont été évalués pour caractériser les morphotypes. L'analyse statistique descriptive a montré que les coefficients de variation vont de $10,3 \%$ (pour le début de floraison) à $72 \%$ (pour le poids des graines par plante). Des valeurs significativement élevées $(\mathrm{CV}>20 \%)$ ont été observées pour 07 caractères sur les 12 analysés. La caractérisation agromorphologique a révélé des différences significatives entre 09 paramètres des morphotypes étudiés. Il est ressorti que les morphotypes $\mathrm{Ne}-10$ et $\mathrm{Ne}-14$ sont plus performants en rendement en grains, avec respectivement 10.8 et 9.6 g/plante. Le morphotype à floraison tardif (Ne-01) a eu le plus faible rendement. Des corrélations fortes ont été observées au niveau des paramètres morphologiques, avec $\mathrm{r}=0,82$ entre la longueur du pétiole et la largeur de la foliole terminale. Une corrélation du même ordre a été obtenue entre l'envergure de la plante et la largeur de la foliole terminale, avec $\mathrm{r}=0,76$. Pour les paramètres du rendement, des fortes corrélations ont été observées entre le poids de graines par plante et le poids de gousses par plante $(r=0,96)$, et entre le même poids de graines et le nombre de gousses par plante ( $\mathrm{r}=$ 0.87). Le dendrogramme a permis de classer les morphotypes en 4 groupes, 
avec les morphotypes $\mathrm{Ne}-14$ et $\mathrm{Ne}-01$ formant chacun deux groupes isolés, les morphotypes $\mathrm{Ne}-04$ et $\mathrm{Ne}-08$ formant le troisième groupe et les 10 autres constituant tous ensemble le quatrième groupe.

Mots-clés: Vigna subterranea, Zone sahélienne, diversité, morphotypes,

Niger

\section{Introduction}

Le Voandzou [Vigna subterranea (L.) Verdc], est généralement cultivé en Afrique sub-saharienne et sahélienne (Konate et al., 2013), où sa production occupe le troisième rang des légumineuses à graines, après l'arachide (Arachis hypogeae L.) et le niébé (Vigna unguiculata L Walp.) (Touré et al., 2013). Cette plante, adaptée à diverses conditions climatiques et écologiques, est principalement cultivée pour ses graines riches en protéines, en glucides et en lipides (Brought et Azam-Ali, 1992 ; Ndiang et al. 2012). Elle est hautement riche en calories ( $387 \mathrm{kcal} / 100 \mathrm{~g}$ ), en protéines, en vitamines et en éléments minéraux (Minka et Bruneteau, 2000 ; Amarteifio et al., 2006 ; Onwubiko et al., 2011, Sévérin et Yao, 2011). En effet, selon Amarteifio et al., 2006, les graines de Voandzou renferment différents éléments minéraux dont le calcium, le magnésium et le potassium. Les protéines qu'elles contiennent ont une forte teneur en lysine et, leur association alimentaire avec les céréales constitue un complément nutritionnel, notamment pour les populations rurales ayant un accès difficile aux protéines animales (Massawe et al., 2005). Par ailleurs, comme toutes les légumineuses, la culture de voandzou contribue à la fertilisation du sol, à travers la fixation symbiotique de l'azote atmosphérique par les bactéries du genre Rhizobium (Mukumbira, 1985, Guye et al., 1998, Sévérin et Yao, 2011, Touré et al., 2013). Certains morphotypes de voandzou résistent bien aux attaques des insectes, aux maladies et aux poches de sécheresse (Mungate, 1997). Ceci confère à la culture de voandzou une bonne capacité d'adaptation aux régions arides d'Afrique sub-saharienne où elle est surtout cultivée par les femmes (Ntundu et al., 2004, Ngwako et al., 2011), en culture pure sur des petites superficies et sans techniques culturales adéquates. En dépit de tous ces avantages, le voandzou fait partie des espèces négligées dans les programmes de sélection variétale (Zerihun, 2009). Sa culture n'a pratiquement d'importance que pour atténuer la souffrance des populations pendant les périodes de soudure. Pourtant, la plante offre d'énormes potentialités d'amélioration, grâce à sa diversité génétique retrouvée dans des milliers d'accessions récoltées à travers le monde (Massawe et al., 2002; Basu et al., 2004 ; Ntundu et al., 2004 ; Djè et al., 2005 ; Ofori et al., 2006 ; Sévérin et Yao, 2011 ; NDiang et al., 2012). Cependant, au Niger très peu d'informations sont actuellement disponibles sur la diversité génétique et agro-physiologique 
de cette espèce qui, pourtant devrait être prise en compte dans la conception des programmes de sélection génétique et agronomique du pays et de la sousrégion ouest-africaine. Les stratégies de sélection envisageables pour l'amélioration génétique de cette espèce demandent d'abord une bonne connaissance de la diversité génétique qui s'est accumulée au sein des variétés traditionnelles, sous les effets des sélections naturelle et humaine. En effet, les morphotypes cultivés par les paysans doivent être investigués et caractérisés pour mieux les discriminer. L'objectif de cette étude est d'évaluer la diversité des morphotypes de voandzou cultivés dans la zone sahélienne du Niger, à travers l'analyse de leurs caractères phénologiques, morphologiques et agronomiques.

\section{Materiel Et Methodes \\ Site d'étude}

L'évaluation des morphotypes a été réalisée au cours de la saison des pluies 2014, à la station expérimentale de l'Institut National de Recherche Agronomique du Niger (INRAN) de N'Dounga ; village situé à environ $8 \mathrm{Km}$ de Kollo $\left(13.30^{\circ} \mathrm{N}\right.$ et $\left.2.34^{\circ} \mathrm{E}\right)$, dans la zone sahélienne. Ce site est caractérisé par une pluviométrie annuelle oscillant entre 983 et $249 \mathrm{~mm}$, avec moyenne générale de $546 \mathrm{~mm}$. La pluviométrie recueillie en 2014 a atteint un cumul de $486 \mathrm{~mm}$.

\section{Matériel Végétal}

Le matériel végétal est composé des graines de 14 morphotypes de Voandzou triées des accessions de la collection effectuée à travers tout le pays du Niger de 2012 à 2013 (Amadou et al., 2014). Sur la base de la couleur et des différents traits sur les graines, les accessions ont été regroupées en quatorze (14) Morphotypes. Ces morphotypes sont ainsi tous caractérisées par la forme, la couleur, la distribution à travers les zones de culture et l'importance dans la collection (Tableau 1, Fig 1).

Tableau 1. Caractéristiques des graines des différents morphotypes du voandzou utilisés

\begin{tabular}{|c|c|c|}
\hline Morphotypes & Caractéristiques & Distribution-importance \\
\hline $\mathrm{Ne}-01$ & $\begin{array}{l}\text { Couleur jaune } \\
\text { Bon goût, très apprécié par les } \\
\text { consommateurs }\end{array}$ & Partout et en grande quantité. Toujours seul \\
\hline $\mathrm{Ne}-02$ & $\begin{array}{l}\text { Noir } \\
\text { Moins apprécié }\end{array}$ & Partout, en mélange et en petite quantité \\
\hline $\mathrm{Ne}-03$ & $\begin{array}{l}\text { Jaune avec œil coloré en forme } \\
\text { de papillon, apprécié par les } \\
\text { consommateurs. }\end{array}$ & Partout et en grande quantité. Le plus souvent seul \\
\hline $\mathrm{Ne}-04$ & $\begin{array}{l}\text { Jaune avec rayures en marron et } \\
\text { œil noir }\end{array}$ & $\begin{array}{l}\text { Un peu partout, en mélange ou seul et en quantité } \\
\text { rarement grande }\end{array}$ \\
\hline $\mathrm{Ne}-05$ & Rouge tâchété de noir & Un peu partout, en mélange et en quantité faible \\
\hline $\mathrm{Ne}-06$ & Rouge sombre & Un peu partout, en mélange et en quantité faible \\
\hline
\end{tabular}




\begin{tabular}{|c|c|c|}
\hline Ne-07 & Rouge clair & Un peu partout, en mélange et en quantité faible \\
\hline Ne-08 & Marron clair & Un peu partout, en mélange et en quantité faible \\
\hline Ne-09 & $\begin{array}{l}\text { Jaune tacheté de noir avec œil en } \\
\text { forme de papillon, coloré en } \\
\text { marron }\end{array}$ & Un peu partout, en mélange et en quantité faible \\
\hline Ne-10 & $\begin{array}{l}\text { Jaune avec petits points marron } \\
\text { sur le tégument, œil marron }\end{array}$ & $\begin{array}{l}\text { Rare, toujours en mélange et en petite quantité, } \\
\text { facilement rencontré dans le Manga }\end{array}$ \\
\hline $\mathrm{Ne}-11$ & $\begin{array}{l}\text { Jaune avec un ou deux traits } \\
\text { marron et œil noir }\end{array}$ & Un peu partout, en mélange et en quantité faible \\
\hline $\mathrm{Ne}-12$ & Grosses graines et rouge claire & Rare et se trouve en petite quantité \\
\hline Ne-13 & $\begin{array}{l}\text { Jaune fortement coloré en noir } \\
\text { avec œil marron }\end{array}$ & Un peu partout, en mélange et en quantité faible \\
\hline $\mathrm{Ne}-14$ & Grise tachetée à œil bronzé & Un peu partout, en mélange et en quantité faible \\
\hline
\end{tabular}
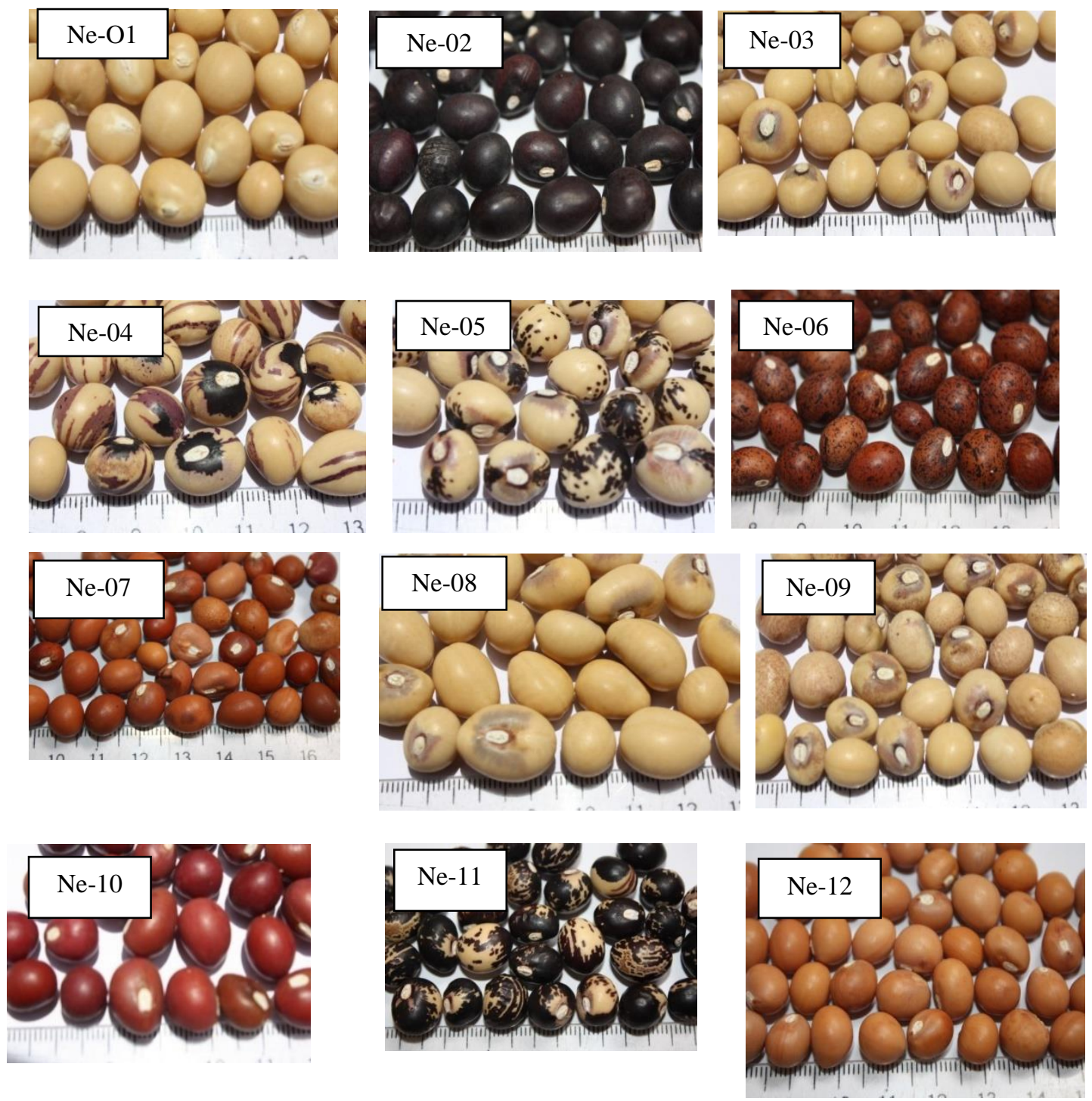

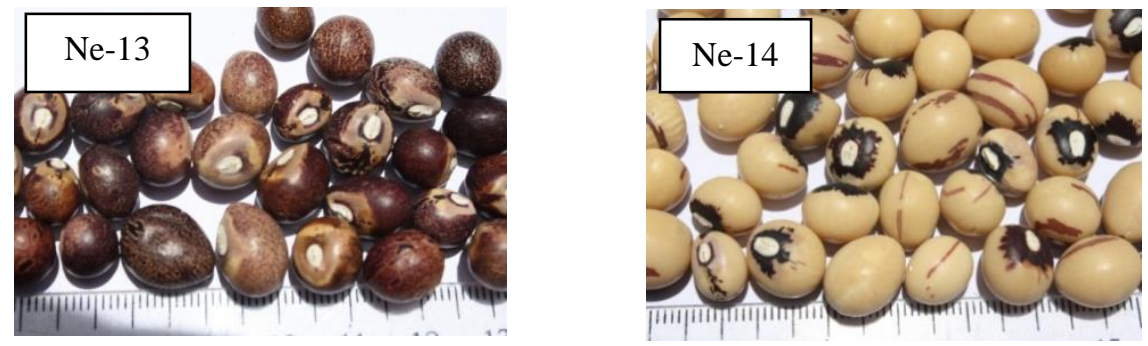

Figure 1 : Photo de graines des 14 morphotypes de voandzou utilisés pour l'étude

\section{Methodes}

\section{Le dispositif expérimental}

L'essai a été installé selon un dispositif en blocs complets randomisés avec quatre répétitions. Chaque répétition est composée d'un bloc de 14 lignes représentant chacune un morphotype. Chaque ligne est composée de 09 poquets équidistants de $0,2 \mathrm{~m}$. La distance entre ligne est de $0,4 \mathrm{~m}$. Chaque bloc mesure $02 \mathrm{~m}$ de largeur $(0,2 \mathrm{~m} \times 10)$ et $06 \mathrm{~m}$ de longueur $(0,4 \mathrm{~m} \times 15)$, soit une superficie de $12 \mathrm{~m}^{2}$. La superficie totale de l'essai est de $48 \mathrm{~m}^{2}\left(12 \mathrm{~m}^{2}\right.$ $\mathrm{x}$ 04). Les graines des 14 morphotypes ont été semées de façon aléatoire par bloc, de sorte qu'ils soient représentés par 09 plantes chacun. Le semis a été effectué le 22 juillet 2014, en conditions pluvieuses.

Douze (12) caractères agro-morphologiques ont été sélectionnés parmi les descripteurs du voandzou, pour caractériser et évaluer les différentes variétés (Tableau 2). Toutes les mesures et observations ont été effectuées sur 5 plantes marquées pour chaque morphotype, en respectant les périodes de collecte des données et les indications de la liste des descripteurs du voandzou (IPGRI/IITA/BAMNET, 2000).

Tableau 2 : Les caractères agro morphologiques utilisées pour évaluer les 14 morphotypes de Voandzou

\begin{tabular}{|c|c|}
\hline \multirow{3}{*}{ Caractères étudiés } & $\begin{array}{l}\text { Paramètres phénologiques : } \\
\text { Nombre de jours entre le semis et la première floraison }(j)\end{array}$ \\
\hline & $\begin{array}{l}\text { Paramètres morphologiques : } \\
\text { Longueur de l'étendard }(\mathrm{mm}) \\
\text { Longueur du pédoncule }(\mathrm{mm}) \\
\text { Nombre de feuilles } \\
\text { Longueur de la foliole terminale }(\mathrm{mm}) \\
\text { Largeur de la foliole terminale }(\mathrm{mm}) \\
\text { Longueur du pétiole }(\mathrm{mm}) \\
\text { Envergure de la plante }(\mathrm{cm}) \\
\text { Hauteur de la plante }(\mathrm{cm}) \\
\end{array}$ \\
\hline & $\begin{array}{l}\text { Paramètres de rendements : } \\
\text { Nombre de gousses par plante } \\
\text { Poids des gousses par plante }(\mathrm{g}) \\
\text { Poids des graines par plante }(\mathrm{g})\end{array}$ \\
\hline
\end{tabular}




\section{Analyse statistique des données}

Les performances des différents morphotypes ont été évaluées en déterminant la moyenne, l'écart type, le coefficient de variation, le minimum et le maximum de chaque caractère. L'analyse de variance a été suivie du test de la plus petite différence significative (LSD, à $\mathrm{P}<0,05)$, pour évaluer la différence entre les morphotypes utilisant le Test de Fisher. Le degré d'interdépendance entre les différents caractères étudiés a été estimé par le coefficient de corrélation de Pearson. Les calculs ont été effectués à l'aide du logiciel XLSTAT version 7.1. Une Classification Ascendante et Hiérarchisée (CAH) a été réalisée avec le logiciel Genstat $10^{\mathrm{e}}$ version.

\section{Resultats Et Discussion Resultats}

L'examen des résultats de l'analyse statistique descriptive indique des écarts importants entre les minima et les maximas des paramètres analysés (Tableau 3). Ce tableau montre que les coefficients de variation sont plus faibles pour les paramètres début de floraison (DF), longueur de l'étendard (LET), Longueur de la foliole terminale (LfT), et largeur de la foliole terminale (lfT) (avec des valeurs respectives de 10,3, 11,5, 15,1\% et 15,2\%) et plus élevés pour les paramètres poids de graines par plante $(\mathrm{Pg})$, poids de gousses par plante (PG) et nombre de gousses par plante (NG), avec des valeurs atteignant respectivement $72 \%, 71,6$ et $66,1 \%$. De façon globale, on observe des coefficients de variation significativement élevées (CV>20\%) pour 7 des 12 caractères analysés. Par ailleurs, on constate que la médiane est proche de la moyenne pour tous les caractères analysés (Tableau 3)

Tableau 3 : Résultat de l'analyse statistique descriptive des paramètres des 14 morphotypes

\begin{tabular}{|lllllll|}
\hline Paramètres & Médiane & Moyenne & Ecart-type & Minimum & Maximum & CV (\%) \\
DF (jour) & 34,0 & 35,1 & 3,6 & 31,0 & 44,0 & 10,3 \\
LfT (mm) & 63,0 & 70,5 & 23,8 & 31,0 & 175,0 & 33,8 \\
IfT (mm) & 23,8 & 26,7 & 4,0 & 20,0 & 52,0 & 15,1 \\
LP (mm) & 120,0 & 122,7 & 18,6 & 80,0 & 195,0 & 15,2 \\
ENV (cm) & 33,0 & 32,6 & 6,9 & 16,0 & 54,0 & 21,1 \\
HP (cm) & 17,0 & 17,8 & 3,4 & 11,0 & 40,0 & 19,0 \\
PG (g) & 9,3 & 10,8 & 7,7 & 0,48 & 47,7 & 71,6 \\
Pg (g) & 6,8 & 7,9 & 5,7 & 0,16 & 33,4 & 72,0 \\
NG & 12,0 & 13,6 & 8,9 & 1,0 & 48,0 & 66,1 \\
LET (mm) & 10,0 & 10,1 & 1,2 & 6,0 & 14,0 & 11,5 \\
Lpd (mm) & 9,0 & 8,8 & 1,9 & 4,0 & 14,0 & 21,6 \\
NF & 58,5 & 60,5 & 22,2 & 17,0 & 169,0 & 36,6 \\
\hline
\end{tabular}

$\boldsymbol{C V}:$ Coefficient de variation ; DF : Début de floraison ; LfT : Longueur de la foliole terminale, lfT : largeur de la foliole terminale $; \boldsymbol{L P}$ : longueur du pétiole $; \boldsymbol{E N V}$ : envergure de la plante $; \boldsymbol{H P}:$ hauteur de la plante ; $\boldsymbol{P G}$ : poids de gousses par plante; $\mathbf{P g}$ : poids de graines par plante; $\mathbf{N G}:$ nombre de gousses par plante ; LET : longueur de l'étendard; Lpd : longueur du pédoncule; NF : nombre de feuilles. 
Sur le plan phénologique, le nombre de jours de croissance avant le début de la première floraison a varié entre $33(\mathrm{Ne}-07, \mathrm{Ne}-02, \mathrm{Ne}-12)$ et 38 jours ( $\mathrm{Ne}-01, \mathrm{Ne}-08)$. Les morphotypes $\mathrm{Ne}-01$ et $\mathrm{Ne}-08$ ont eu des dates de début de floraison non statistiquement différentes entre elles, mais significativement plus tardif que celles des autres morphotypes (Tableau 4). L'analyse des résultats de ce tableau montre que le caractère morphologique portant sur la longueur de la foliole terminale (LfT) a varié entre 55,8 mm (Ne$05)$ et $111 \mathrm{~mm}(\mathrm{Ne}-14)$, avec une différence significative observée entre les différents morphotypes $\left(\mathrm{F}=25,45^{* *}\right)$. Les autres caractères morphologiques comme la largeur de la foliole terminale (lfT), la longueur du pétiole (LP), l'envergure de la plante (ENV), la hauteur de la plante (HP) et la longueur de l'étendard (LET) ont aussi montré des différences significatives entre les différents morphotypes. Cependant, la longueur du pédoncule (Lpd) a varié entre $7.9 \mathrm{~mm}(\mathrm{Ne}-04)$ à $9.4 \mathrm{~mm}(\mathrm{Ne}-01)$ sans montrer une différence statistiquement significative $(\mathrm{F}=1.09)$ entre les différents morphotypes (Tableau 2). De même, le caractère portant sur le nombre de feuilles (NF) a varié entre $450(\mathrm{Ne}-14)$ et 68 (Ne-02) sans montrer une différence significative entre les différents morphotypes (Fisher $=1.00)$.

Tableau 4 : Moyennes des neuf (9) paramètres morpho-phénologiques des 14 morphotypes de voandzou

\begin{tabular}{|c|c|c|c|c|c|c|c|c|c|}
\hline Morphotypes & $\begin{array}{l}\text { DF } \\
\text { (j) }\end{array}$ & $\begin{array}{l}\text { LfT } \\
(\mathrm{mm})\end{array}$ & $\begin{array}{l}\text { IfT } \\
(\mathrm{mm})\end{array}$ & $\begin{array}{l}\mathbf{L P} \\
(\mathrm{mm})\end{array}$ & $\begin{array}{l}\text { ENV } \\
(\mathrm{cm})\end{array}$ & $\begin{array}{l}\mathbf{H P} \\
(\mathrm{cm})\end{array}$ & $\begin{array}{l}\text { LET } \\
(\mathrm{mm})\end{array}$ & $\begin{array}{l}\text { Lpd } \\
(\mathrm{mm})\end{array}$ & NF \\
\hline $\mathrm{Ne}-01$ & $38,4^{\mathrm{a}}$ & $58,2^{c}$ & $25,4^{b}$ & $124,8^{\mathrm{c}}$ & $30,8^{c}$ & $16,1^{\mathrm{e}}$ & $10,5^{b}$ & $9,4^{\mathrm{a}}$ & $56,3^{\mathrm{a}}$ \\
\hline $\mathrm{Ne}-02$ & $33,4^{\mathrm{b}}$ & $59,3^{\mathrm{c}}$ & $26,1^{\mathrm{b}}$ & $113,8^{\mathrm{c}}$ & $32,2^{\mathrm{c}}$ & $17,3^{\mathrm{e}}$ & $9,7^{c}$ & $8,9^{\mathrm{a}}$ & $68,2^{\mathrm{a}}$ \\
\hline $\mathrm{Ne}-03$ & $35,8^{\mathrm{ab}}$ & $60,7^{\mathrm{c}}$ & $27,1^{\mathrm{b}}$ & $126,3^{b c}$ & $33,2^{b}$ & $18,2^{\mathrm{de}}$ & $10,3^{\mathrm{bc}}$ & $9,1^{\mathrm{a}}$ & $64,9^{\mathrm{a}}$ \\
\hline $\mathrm{Ne}-04$ & $34,8^{\mathrm{b}}$ & $65,8^{c}$ & $28,2^{\mathrm{b}}$ & $133,8^{\mathrm{ab}}$ & $36,0^{\mathrm{a}}$ & $20,0^{\mathrm{ab}}$ & $9,9 \mathrm{c}$ & $7,9^{\mathrm{a}}$ & $57,1^{\mathrm{a}}$ \\
\hline $\mathrm{Ne}-05$ & $35,4^{\mathrm{b}}$ & $55,8^{\mathrm{c}}$ & $24,8^{b}$ & $111,0^{\mathrm{c}}$ & $30,9^{c}$ & $16,4^{\mathrm{e}}$ & $10,1^{\mathrm{c}}$ & $8,9^{\mathrm{a}}$ & $59,6^{\mathrm{a}}$ \\
\hline $\mathrm{Ne}-06$ & $34,8^{\mathrm{b}}$ & $56,5^{\mathrm{c}}$ & $24,6^{\mathrm{b}}$ & $112,3^{\mathrm{c}}$ & $29,0^{\mathrm{c}}$ & $17,0^{\mathrm{e}}$ & $10,2^{\mathrm{c}}$ & $8,4^{\mathrm{a}}$ & $60,9^{\mathrm{a}}$ \\
\hline $\mathrm{Ne}-07$ & $33,3^{\mathrm{b}}$ & $56,3^{c}$ & $26,0^{\mathrm{b}}$ & $110,8^{c}$ & $30,0^{c}$ & $16,1^{\mathrm{e}}$ & $9,9^{\mathrm{c}}$ & $8,5^{\mathrm{a}}$ & $59,9^{\mathrm{a}}$ \\
\hline $\mathrm{Ne}-08$ & $38,3^{\mathrm{a}}$ & $66,9^{\mathrm{bc}}$ & $30,1^{\mathrm{a}}$ & $145,0^{\mathrm{a}}$ & $35,6^{\mathrm{a}}$ & $19,9^{\mathrm{b}}$ & $10,7^{\mathrm{a}}$ & $8,9^{\mathrm{a}}$ & $53,9^{\mathrm{a}}$ \\
\hline $\mathrm{Ne}-09$ & $34,6^{\mathrm{b}}$ & $59,7^{\mathrm{c}}$ & $26,4^{\mathrm{b}}$ & $124,5^{\mathrm{c}}$ & $35,9^{a}$ & $19,2^{\mathrm{cd}}$ & $10,7^{\mathrm{a}}$ & $9,3^{\mathrm{a}}$ & $65,6^{\mathrm{a}}$ \\
\hline $\mathrm{Ne}-10$ & $35,5^{\mathrm{b}}$ & $64,8^{c}$ & $28,2^{\mathrm{ab}}$ & $142,8^{\mathrm{a}}$ & $33,8^{\mathrm{ab}}$ & $21,2^{\mathrm{a}}$ & $10,5^{\mathrm{b}}$ & $8,8^{\mathrm{a}}$ & $60,8^{\mathrm{a}}$ \\
\hline $\mathrm{Ne}-11$ & $34,4^{\mathrm{b}}$ & $73,5^{b}$ & $25,6^{\mathrm{b}}$ & $120,0^{c}$ & $32,0^{\mathrm{c}}$ & $16,9^{\mathrm{e}}$ & $9,5^{\mathrm{c}}$ & $9,2^{\mathrm{a}}$ & $61,9^{\mathrm{a}}$ \\
\hline $\mathrm{Ne}-12$ & $33,4^{\mathrm{b}}$ & $96,5^{\mathrm{b}}$ & $26,7^{b}$ & $111,3^{\mathrm{c}}$ & $30,8^{c}$ & $15,2^{\mathrm{e}}$ & $10,0^{\mathrm{c}}$ & $9,3^{\mathrm{a}}$ & $65,7^{\mathrm{a}}$ \\
\hline $\mathrm{Ne}-13$ & $35,0^{\mathrm{b}}$ & $99,9^{\mathrm{a}}$ & $26,6^{\mathrm{b}}$ & $116,5^{\mathrm{c}}$ & $30,9^{\mathrm{c}}$ & $15,8^{\mathrm{e}}$ & $10,5^{\mathrm{ab}}$ & $8,3^{\mathrm{a}}$ & $62,4^{\mathrm{a}}$ \\
\hline $\mathrm{Ne}-14$ & $35,2^{\mathrm{b}}$ & $111,4^{\mathrm{a}}$ & $27,9^{\mathrm{b}}$ & $125,3^{\mathrm{c}}$ & $35,5^{\mathrm{a}}$ & $19,5^{\mathrm{bc}}$ & $9,2^{\mathrm{c}}$ & $8,5^{\mathrm{a}}$ & $49,9^{\mathrm{a}}$ \\
\hline Moyenne & 35,14 & 70,50 & 26,69 & 122,69 & 32,62 & 17,78 & 10,10 & 8,77 & 60,56 \\
\hline Fisher & $4,17 * *$ & $25,45^{* *}$ & $3,03 * *$ & $11,01 * *$ & $2,61^{* *}$ & $8,31 * *$ & $3,29 * *$ & $1,09^{\mathrm{ns}}$ & $1,00^{\text {ns }}$ \\
\hline
\end{tabular}

** : très significative ; * significative ; $\boldsymbol{n s}:$ non significative ; DF : Début de floraison; LfT : Longueur de la foliole terminale, IfT : largeur de la foliole terminale ; LP : longueur du pétiole ; $\boldsymbol{E N V}$ : envergure de la plante; HP : hauteur de la plante; $\mathbf{L E T}$ : longueur de l'étendard; Lpd : longueur du pédoncule ; NF : nombre de feuilles.

L'évaluation des paramètres liés au rendement résumée dans le tableau 5 montre que le nombre de gousses par plante a varié entre $6(\mathrm{Ne}-01)$ et 18 (Ne-06). Les morphotypes $\mathrm{Ne}-06, \mathrm{Ne}-07$ et $\mathrm{Ne}-10$ ont donné les plus grands 
nombres de gousses par plante, avec respectivement 18, 17 et 16 gousses (Tableau 5). Ce tableau montre qu'en termes de production de gousses, ces morphotypes sont significativement différents des autres. Le poids de gousses par plante il a varié entre 4,2 $\mathrm{g}(\mathrm{Ne}-01)$ et 14,8 g (Ne-10) et celui de graines par plante entre 3,3 g (Ne-01) et 10,8 g (Ne-10). Les morphotypes $\mathrm{Ne}-10, \mathrm{Ne}-$ $14, \mathrm{Ne}-03, \mathrm{Ne}-06$ et $\mathrm{Ne}-07$ ont montré les poids de gousses par plante les plus élevés, avec des valeurs respectives de $14.8 ; 13.6 ; 12.5 ; 11.7 ; 11.4$ g/plante. En termes de production de graines, les morphotypes $\mathrm{Ne}-10, \mathrm{Ne}-14$ et $\mathrm{Ne}-07$ ont été les plus performants, avec respectivement $10.8 ; 9.6$ et 9.1 graines/plante. L'analyse de la variance a montré des différences significatives entre les poids moyens des gousses et des graines des différents morphotypes, avec des valeurs de $\mathrm{F}$ de $1.76^{*}$ et $1,44^{*}$, respectivement (Tableau 5).

Tableau 5 : Nombre de gousses et poids moyens en gousses et en graines des 14 morphotypes de voandzou

\begin{tabular}{|c|c|c|c|}
\hline Morphotypes & $\begin{array}{c}\text { Nombre de gousses } \\
\text { par plante }\end{array}$ & $\begin{array}{c}\text { Poids de gousses par } \\
\text { plante }(\mathrm{g})\end{array}$ & $\begin{array}{c}\text { Poids de graines par } \\
\text { plante }(\mathrm{g})\end{array}$ \\
\hline $\mathrm{Ne}-01$ & $5,8^{\mathrm{b}}$ & $4,2^{\mathrm{b}}$ & $3,3^{\mathrm{b}}$ \\
$\mathrm{Ne}-02$ & $13,1^{\mathrm{b}}$ & $8,9^{\mathrm{b}}$ & $7,2^{\mathrm{b}}$ \\
$\mathrm{Ne}-03$ & $14,3^{\mathrm{b}}$ & $12,5^{\mathrm{ab}}$ & $8,4^{\mathrm{b}}$ \\
$\mathrm{Ne}-04$ & $9,3^{\mathrm{b}}$ & $9,1^{\mathrm{b}}$ & $6,2^{\mathrm{b}}$ \\
$\mathrm{Ne}-05$ & $14,9^{\mathrm{b}}$ & $10,2^{\mathrm{b}}$ & $7,4^{\mathrm{b}}$ \\
$\mathrm{Ne}-06$ & $17,9^{\mathrm{a}}$ & $11,7^{\mathrm{b}}$ & $8,8^{\mathrm{b}}$ \\
$\mathrm{Ne}-07$ & $17,3^{\mathrm{a}}$ & $11,4^{\mathrm{b}}$ & $9,1^{\mathrm{b}}$ \\
$\mathrm{Ne}-08$ & $8,9^{\mathrm{b}}$ & $9,9^{\mathrm{b}}$ & $7,1^{\mathrm{b}}$ \\
$\mathrm{Ne}-09$ & $12,9^{\mathrm{b}}$ & $12,4^{\mathrm{b}}$ & $8,0^{\mathrm{b}}$ \\
$\mathrm{Ne}-10$ & $16,4^{\mathrm{a}}$ & $14,8^{\mathrm{a}}$ & $10,8^{\mathrm{a}}$ \\
$\mathrm{Ne}-11$ & $14,3^{\mathrm{b}}$ & $10,6^{\mathrm{b}}$ & $7,9^{\mathrm{b}}$ \\
$\mathrm{Ne}-12$ & $14,3^{\mathrm{b}}$ & $10,4^{\mathrm{b}}$ & $7,9^{\mathrm{b}}$ \\
$\mathrm{Ne}-13$ & $11,6^{\mathrm{b}}$ & $9,5^{\mathrm{b}}$ & $6,9^{\mathrm{b}}$ \\
$\mathrm{Ne}-14$ & $15,6^{\mathrm{ab}}$ & $13,6^{\mathrm{a}}$ & $9,6^{\mathrm{b}}$ \\
\hline Moyenne & 13,58 & 10,81 & 7,92 \\
\hline Fisher & $2,58^{* *}$ & $1,76^{*}$ & $1,44^{\mathrm{b}}$ \\
\hline \multicolumn{2}{|c|}{ : très significative $; *$ significative } \\
\hline
\end{tabular}

Afin d'identifier les relations qui peuvent exister entre les 12 caractères étudiés, une matrice de corrélation (coefficient de Pearson) a été réalisée (Tableau 6). Cette matrice montre que la date de début floraison (DF) est positivement corrélée avec la longueur des pétioles (LP) des feuilles (avec $r=$ 0,62 ) et dans une moindre mesure avec la longueur de l'étendard (LET), avec $\mathrm{r}=0,50$. Par contre, elle a eu une corrélation négative entre le nombre de gousses par plante (NG) et le nombre de feuilles par plante (NF), avec des coefficients de corrélation (r) de $-0,65$ et $-0,56$, respectivement. Quant à la longueur de la foliole terminale (LfT), elle n'a eu aucune corrélation avec les paramètres étudiés, de même que la longueur du pédoncule (Lpd). Cependant, la largeur de la foliole terminale (lfT) a eu une corrélation très significative 
avec la longueur du pétiole (LP), l'envergue (ENV) et la hauteur de la plante (HP), avec des coefficients corrélation (r) de $0,83^{* *}, 0,76^{* *}$ et $0,74 *$, respectivement. De même, la longueur du pétiole est significativement corrélée avec l'envergure et la hauteur de la plante, avec des coefficients de corrélation respectifs de 0,75 et 0,52 . Par ailleurs, de très fortes corrélations ont été obtenues entre le poids des graines et le poids des gousses par plante $\left(\mathrm{r}=0.96^{* * *}\right)$, entre le poids de graines et le nombre de gousses par plante $\left(\mathrm{r}=0.87^{* *}\right)$ et entre le poids des gousses et le nombre de gousses par plante $\left(0.76^{* *}\right)$.

Tableau 6: Matrice de corrélation entre les 12 paramètres de croissances et de production étudiés pour le Voandzou dans la zone sahélienne du Niger

\begin{tabular}{|c|c|c|c|c|c|c|c|c|c|c|c|c|}
\hline & DF & LfT & lfT & LP & ENV & $\mathrm{HP}$ & PG & $\mathrm{Pg}$ & $\mathrm{NG}$ & LET & Lpd & $\mathrm{NF}$ \\
\hline DF & 1 & & & & & & & & & & & \\
\hline LfT & $-0,14$ & 1 & & & & & & & & & & \\
\hline lfT & 0,34 & $\begin{array}{l}0,29 \\
-\end{array}$ & 1 & & & & & & & & & \\
\hline LP & $0,62 *$ & 0,04 & $0,83 * *$ & 1 & & & & & & & & \\
\hline ENV & 0,24 & $\begin{array}{l}0,14 \\
-\end{array}$ & $0,76 * *$ & $0,75 *$ & 1 & & & & & & & \\
\hline HP & 0,27 & 0,05 & $0,74 *$ & $0,52 *$ & $0,85 * *$ & 1 & & & & & & \\
\hline PG & $-0,37$ & 0,20 & 0,29 & 0,19 & 0,33 & $0,52 *$ & 1 & & & & & \\
\hline $\mathrm{Pg}$ & $-0,47$ & 0,17 & 0,18 & 0,05 & 0,11 & 0,38 & $0,96 * *$ & 1 & & & & \\
\hline NG & $-0,65$ & $\begin{array}{l}0,04 \\
-\end{array}$ & $-0,29$ & $-0,40$ & $-0,30$ & $-0,04$ & $0,76 * *$ & $0,87 * *$ & $\begin{array}{l}1 \\
-\end{array}$ & & & \\
\hline LET & $\mathbf{0 , 5 0}$ & $\begin{array}{l}0,36 \\
-\end{array}$ & 0,19 & 0,37 & 0,04 & 0,13 & $-0,15$ & $-0,23$ & $\begin{array}{l}0,37 \\
-\end{array}$ & 1 & & \\
\hline Lpd & 0,23 & $\begin{array}{l}0,15 \\
-\end{array}$ & $-0,11$ & 0,05 & $-0,01$ & $-0,13$ & $-0,15$ & $-0,19$ & 0,21 & 0,21 & 1 & \\
\hline $\mathrm{NF}$ & $-0,56$ & 0,26 & $-0,39$ & $-0,43$ & $-0,32$ & $-0,33$ & 0,05 & 0,08 & 0,24 & 0,14 & 0,34 & 1 \\
\hline
\end{tabular}

Une analyse multi variée a permis de construire un dendrogramme permettant de voir le niveau de troncature entre les différents morphotypes. Le dendrogramme a permis de discriminer 4 groupes de morphotypes. Le morphotype $\mathrm{Ne}-14$ forme à lui seul le premier groupe, les morphotypes $\mathrm{Ne}$ $10 ; \mathrm{Ne}-13$; Ne-07 ; Ne-06 ; Ne-05 ; Ne-12 ; Ne-02 ; Ne-11 ; Ne-09 et Ne-03 forment le deuxième groupe, les morphotypes $\mathrm{Ne}-04$ et $\mathrm{Ne}-08$ forment le troisième groupe et le morphotype $\mathrm{Ne}-01$ forme le quatrième groupe, à la troncature 0,875 . Il faut donc noter l'isolement des morphotypes $\mathrm{Ne}-01$ et $\mathrm{Ne}-$ 14 des autres morphotypes (Figure 2). 


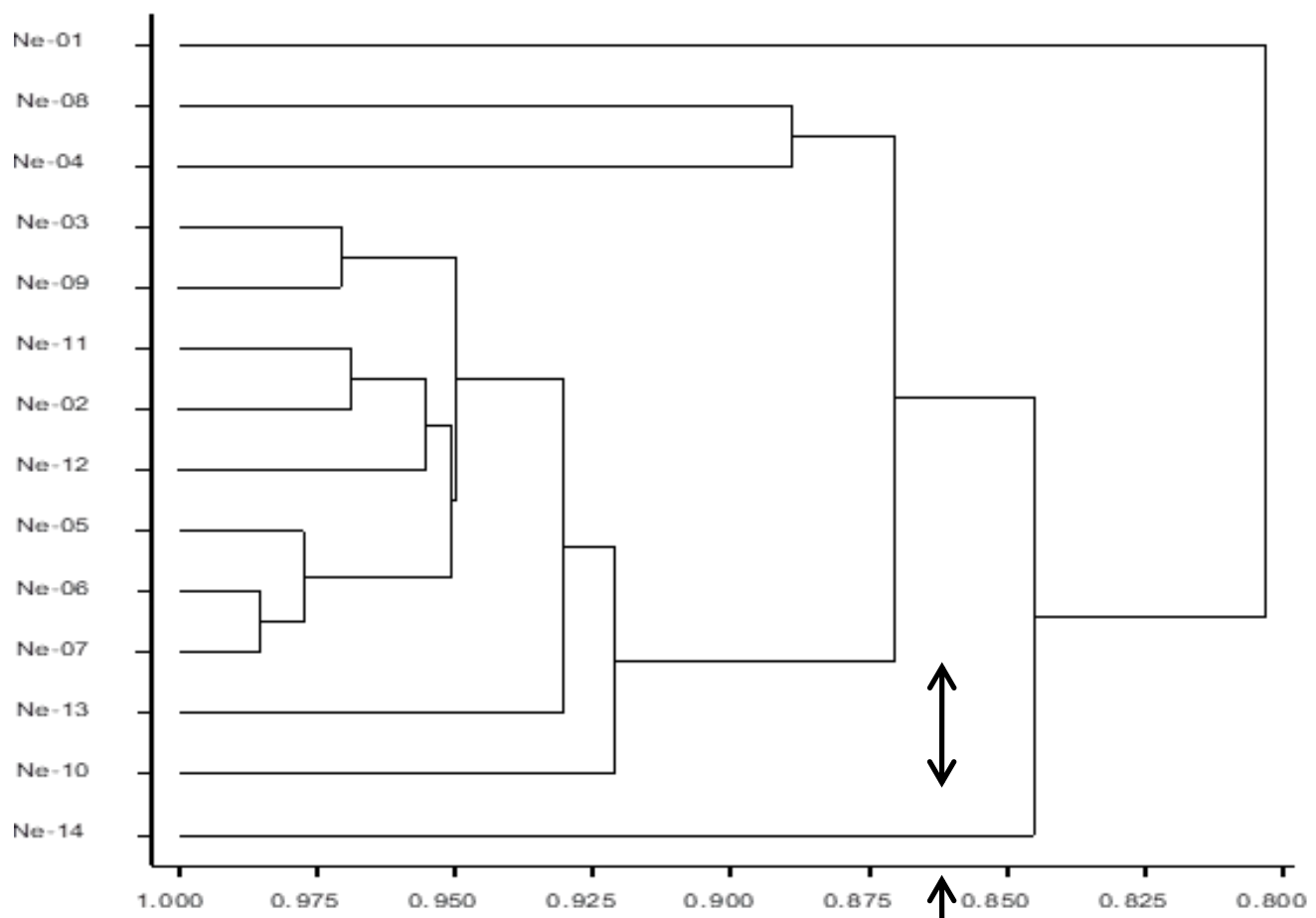

Figure 2 : Dendrogramme issu de la classification ascendante hiévirchisée selon la méthode de Ward, montrant les relations phylogénétiques entre les 14 morphotypes de voandzou

\section{Discussion}

L'analyse des données a permis de caractériser les différents morphotypes de voandzou étudiés. En effet, l'analyse de la variance a permis de mettre en évidence les différences qui existent entre les morphotypes, à travers les paramètres étudiés. Cette caractérisation agro-morphologique des morphotypes de voandzou est une étape très importante dans l'évaluation de la diversité génétique de cette culture, dans la zone sahélienne du Niger. En effet, la méthodologie de l'étude a permis de mesurer les paramètres de croissance et de développement des différents morphotypes de voandzou testés et d'évaluer les composantes de leurs rendements.

Les résultats obtenus ont montré des différences significatives entre les minima et les maximas des paramètres étudiés, avec des coefficients de variation particulièrement plus faibles pour le début floraison, la longueur de l'étendard, la longueur de la foliole terminale, et largeur de la foliole terminale. Les plus grands coefficients de variation ont été observés notamment au niveau des paramètres liés au rendement comme le poids de graines, le poids de gousses et le nombre de gousses par plante. Ce résultat montre qu'il y a une forte hétérogénéité au sein des différents morphotypes de voandzou, du point de vue rendement. Parmi les 12 caractères étudiés, 7 ont eu des coefficients de variation significativement élevés, mais un coefficient de variation $(\mathrm{CV})$ 
obtenu pour le nombre de feuilles par plante $(36,6 \%)$ nettement inférieur à celui obtenu par Amadou et al., 2015 (48,45\%), dans une étude similaire utilisant les mêmes morphotypes, mais dans conditions climatiques différentes (en zone soudanienne du Niger). Aussi, Sévérin et Yao, 2011 ont obtenu un CV encore plus grand $(62,3 \%)$, dans leur étude sur la variabilité morphologique et agronomique des variétés traditionnelles de voandzou [Vigna subterranea (L.) verdc.] issus de 101 accessions. Par contre, le coefficient de variation obtenu entre les feuilles des différents morphotypes est supérieur à celui obtenu par Massawe et al., 2005 (31.6\%) ayant étudié six (6) accessions. Cependant, le CV du nombre de gousses par plante $(66,1 \%)$ obtenu dans cette étude pour la zone sahélienne est plus élevé que celui qui a été obtenu en Côte d'Ivoire par Bonny et al., 2011 (47,7\%). Ces mêmes morphotypes, cultivés en zone soudanienne ont montré un faible coefficient de variation (59.6\%) par rapport à leur culture en zone sahélienne (Amadou et al., 2015).

Cette étude a montré que le début de la floraison se situe entre le $33^{\text {ème }}$ jour après semis (chez les morphotypes $\mathrm{Ne}-07, \mathrm{Ne}-02$ et $\mathrm{Ne}-12$ ) et le $38^{\text {ème }}$ jour après semis (chez les morphotypes Ne-08 et Ne-01), soit un intervalle de 5 jours. Cependant, Ndiang et al., 2012 ont trouvé un intervalle plus grand de 17 jours se situant entre le $38^{\text {ème }}$ et le $55^{\text {ème }}$ jour après semis, dans leur étude sur la variabilité morphologique de voandzou au Cameroun. De même Djé et al. (2005), ont observé un intervalle de 15 jours (entre le $41^{\text {ème }}$ et le $56^{\text {ème }}$ jour après semis), dans leur étude sur l'observation préliminaire de la variabilité entre quelques morphotypes de voandzou de Côte d'Ivoire. Ces différences pourraient s'expliquer par la diversité des comportements génétiques des morphotypes étudiés et les variations des conditions climatiques des zones de culture. Ceci peut se justifier à travers la différence significative observée, pour le caractère phénologique de début de floraison, entre les différents morphotypes étudiés dans la zone sahélienne; alors que les mêmes morphotypes évalués dans la zone soudanienne n’ont montré aucune différence significative (Amadou et al. 2015). La hauteur de la plante a atteint un minimum de 15,2 cm (chez le morphotype Ne-12) et un maximum de 21.2 cm (chez le morphotype $\mathrm{Ne}-10)$. Ces valeurs sont plus élevées que celles trouvées par Touré et al., 2013 et Amadou et al. 2015 (14 et 19,6 cm), pour les mêmes morphotypes. Par contre, elles sont inférieures à celles obtenues par Djè et al. 2005, (20 à $29 \mathrm{~cm}$ ) en Côte d'Ivoire et par Ndiang et al. 2012, (20 à $38 \mathrm{~cm}$ ) au Cameroun.

Il a été constaté que l'analyse des caractères liés au rendement a montré une certaine variabilité entre les différents morphotypes étudiés, notamment en ce qui concerne le nombre de gousse a varié entre 6 et 18 gousses par plante. Ce résultat est plus au moins similaire à celui obtenu par Amadou et al. (2015) ayant évalué ces morphotypes à la même période, mais nettement inférieur à 
ceux obtenus par Edjé et Sesay, (2004), Ndiang et al., en (2012) et Touré et al., (2013). Les morphotypes $\mathrm{Ne}-10$ et Ne-14 ont eu des nombres de gousses, des poids gousses des rendements en grain par plante statistiquement plus élevés que ceux des autres morphotypes. La matrice de corrélation (coefficient de Pearson) réalisée entre les différents caractères a montré que les corrélations les plus significatives ont été observées entre le poids des graines par plante et le poids des gousses $(r=0.96)$, entre le même poids des graines et le nombre des gousses $(r=0.87)$ et entre la hauteur de la plante et l'envergure $(\mathrm{r}=0.85)$. Ces résultats corroborent ceux d'Ouedrago et al. (2008) qui ont rapporté, que les caractères comme le nombre de gousses par plante, le nombre de grains par gousse et le poids des 100 grains sont positivement corrélés avec le rendement par plante $(\mathrm{Pg})$, chez le voandzou. Par contre, une corrélation négative a été observé entre le début de la floraison (DF) et le rendement en grain par plante ; ceci du fait que les morphotypes à long cycle végétatif produisent généralement moins dans la zone sahélienne, comme révélé par les caractéristiques du morphotype $\mathrm{Ne}-01$ ayant eu la date de floraison la plus tardive (38.4 jours après semis) et le rendement par plante le plus faible $(3.3 \mathrm{~g} / \mathrm{plante})$.

L'ensemble de ces résultats montrent l'existence d'une diversité importante au sein de l'espèce. Cette importante variabilité phénotypique pourrait résulter non seulement de l'expression d'une forte hétérogénéité génotypique, mais aussi de l'influence des facteurs environnementaux. En effet, de nombreux travaux ont montré que la longueur du jour (Harris et Azam-ali, 1993 ; Linnemann et al., 1995 ; Brink, 1999), la température (Linnemann et Craufurd, 1994 ; Brink, 1998) et 1'humidité (Collinson et al., 1996) entraînent des effets variables sur le développement végétatif et physiologique du voandzou et peuvent impacter ses rendements. Selon les différentes caractéristiques morphologiques et agromorphologiques étudiés, les morphotypes ont montré une certaine diversité et l'analyse du dendrogramme a montré un regroupement des morphotypes en 4 groupes, avec les morphotypes $\mathrm{Ne}-14$ et $\mathrm{Ne}-01$ formant chacun un groupe, les morphotypes $\mathrm{Ne}-04$ et $\mathrm{Ne}-08$ forment un troisième et les 10 autres formant un quatrième groupe. Ces différents groupes sont très proches sur tous les caractères étudiés. Les morphotypes des groupes 1 et 2 sont en général caractérisés par une floraison tardive avec des faibles rendements tandis que les $3^{\mathrm{e}}$ et $4^{\mathrm{e}}$ groupes présentent des rendements en grains par plante et parfois même avec une différence statiquement significative avec les morphotypes à floraison tardive (entre $\mathrm{Ne}-14$ et $\mathrm{Ne}-01$ ).

\section{Conclusion}

Une étude de la variabilité morphologique et agromorphologiques de quatorze morphotypes de voandzou a été menée dans la zone sahélienne du 
Niger, afin d'évaluer les caractères agro morphologiques chez cette espèce. La caractérisation agro morphologique a montré une différence significative entre les 14 morphotypes de voandzou, notamment pour les paramètres de croissance végétative et de rendement. En effet, parmi les douze paramètres étudiés, 10 peuvent être utilisés pour faire la distinction entre les différents morphotypes ; ceux liés à la longueur du pédoncule et au nombre de feuille n'ayant montré aucune différence significative entre les différents morphotypes. Il faut relever que les morphotypes $\mathrm{Ne}-07, \mathrm{Ne}-10$ et $\mathrm{Ne}-14$ sont plus performants en rendement grains par plante. En raison de leur forte capacité productive, ces morphotypes peuvent être sélectionnés à des fins alimentaires ou être utilisés pour l'amélioration de la production au Niger et dans le Sahel. Par contre les morphotypes Ne-08 et Ne-01 à floraison tardive ont des faibles rendements en grain. Ceci indique que ces morphotypes ne sont pas favorables à une quelconque sélection de voandzou en zone sahélienne.

\section{Remerciements}

Les auteurs remercient les autorités rectorales de l'Université Abdou Moumouni pour leur soutien financier qui a permis de mener cette étude. Ils apprécient la collaboration du personnel de la station de recherche de Kollo qui a activement participé à la conduite de l'essai agronomique. Ils remercient également le Directeur Général de l'Institut National de la Recherche Agronomique du Niger (INRAN) pour avoir autorisé la conduite de l'essai dans son champ d'expérimentation à Kollo.

\section{References:}

1. Amadou H.I., Yacoubou B., Zoubeirou A.M., Ali D., Ibrahim M.B. (2014). Diagnostic participatif de la diversité de morphotypes et des connaissances locales en matière de culture du Voandzou (Vigna Subterranea L.) au Niger. International Journal of Innovation and Applied Studies; 9(4): 1915-1925.

2. Amadou H.I., Doumma A., Katsileros A., Zoubeirou A.M., Nourou S.S.M. (2015). "Agro-morphological variability in fourteen bambara groundnut (Vigna subterranea (L.) Verdc.) morphotypes cultivated in Niger. Scholars. Academic Journal of Biosciences. 3(9):774-781

3. Amarteifio J.O., Tibe O., Njogu R.M. (2006). The mineral composition of bambara groundnut (Vigna subterranea (L.) Verdc) grown in Southern Africa. African Journal of Biotechnology 5: 24082411.,

4. Basu S., Roberts J., Mithen R., Azam-Ali S., Pasquet R. (2004). The genetics of Bambara groundnut and the construction of a genetic linkage map. In New directions for a diverse planet: Proceedings of the 4th International Crop Science Congress Brisbane, Australia. 7p. 
5. Brink M. (1999). Development, growth and dry matter partitioning in bambara groundnut (Vigna subterranea) as influenced by photoperiod and shading. Journal of Agricultural Sciences 133, 159-166 p.

6. Brink M., (1998). Matching crops and environments: quantifying photothermal influences on reproductive development in bambara groundnut (Vigna subterranea (L.) Verdc). PhD Thesis, Wageningen Agricultural University, $161 \mathrm{pp}$.

7. Brough S.H., Azam-Ali S.N. (1992). The effect of soil moisture on the proximate composition of Bambara groundnut [Vigna subterranea (L.) Verdc.]. Journal of Science Food and Agriculture 60: 197-203

8. Collinson S.T., Azam-Ali S.N., Chavula K.M., Hodson D.A. (1996). Growth, development and yield of bambara groundnut (Vigna subterranea) in response to soil moisture. Journal of Agricultural Sciences 126, 307-318 p.

9. Djè Y, Bonny BS, Zoro Bi IA, 2005. Observations préliminaires de la variabilité entre quelques morphotypes de voandzou (Vigna subterranea (L.) Verdc., Fabaceae) de Côte d'Ivoire. Biotechnologie, Agronomie, Société et Environnement Volume 9 : 249-258.

10. Edjé OT, Sesay A, 2004. Effects of seed source on performance and yield of Bambara groundnut [Vigna subterranea (L.) Verdc.] landraces. In: Proceeding of the International bambara Groundnut Symposium" European Union Framework Programme 5 Botswana College of Agriculture, Botswana 8 -12 September 2003. 141-152 pp.

11. Gueye M., James E.K., Kierans M. and Sprent J.L. (1998). The development and structure of root nodules on Bambara groundnut (Vigna subterranea L.), W. Journal of Microbiology and. Biotechnology (14) 177-184 pp

12. Harris D., Azam-Ali S.N. (1993). Implications of day length sensitivity in bambara groundnut (Vigna subterranea) for production in Botswana. Agricultural Sciences. 120, 75-78 p.

13. IPGRI, IITA, BAMNET, (2000). Descripteurs du pois bambara (Vigna subterranea). Institut international des ressources phytogénétiques, Rome, Italie ; Institut International d'Agriculture Tropicale, Ibadan, Nigeria ; Réseau International de pois bambara, Allemagne. 59 p,

14. Konaté S., Mongomaké K., Edmond K. K., Michel Z., Justin Y. K. (2013). Induction et prolifération de cals à partir de l'axe embryonnaire du Voandzou [Vigna subterranea (L.) Verdc. Fabaceae] : effet de la segmentation de l'explant, des phytohormones, de la source de carbone et du génotype. Afrique SCIENCE 09(1) (2013) 140 - 150,

15. Linnemann A., Craufurd P. (1994). Effects of temperature and photoperiod on phenological development in three genotypes of 
bambara groundnut (Vigna subterranea). Annales of Botanic 74, 675$681 \mathrm{p}$.

16. Linnemann A.R., Westphal E., Wessel M. (1995). Photoperiod regulation of development and growth in bambara groundnut (Vigna subterranea). Field Crops Research 40, 39-47 p.

17. Massawe F.J., Mwale S.S., Azam-Ali S.N., Roberts J.A. (2005). Breeding in Bambara groundnut (Vigna subterranea L. Verdc.): strategic considerations. African Journal of Biotechnology, 4(6): 463471.

18. Massawe F.J., Dickinson M., Roberts J.A., Azam-ali S.N. (2002). Genetic diversity in bambara groundnut (Vigna subterranea (L.) Verdc.) landraces revealed by AFLP markers. Genome 45: 1175-1180.

19. Minka S.R., Bruneteau M. (2000). Partial chemical composition of bambara pea (Vigna subterranea (L.) Verdc). Food Chemistry, 68: 273-276.

20. Mungate D. (1997). Bambara groundnut [Vigna subterranea (L.) Verdc.]. In: Heller J, Begemann F., Mushonga J., eds. Promoting the conservation and use of underutilized and neglected crops. 9. Proceedings of the workshop on Conservation and Improvement of Bambara Groundnut (Vigna subterranea (L.) Verdc.), 14-16 November 1995, Harare, Zimbabwe.

21. Nding Z., BELL J.M., Missoup A. D., Fokam P. E., Amougou A. (2012). Etude de la variabilité morphologique de voandzou (Vigna Subterranea) au Cameroun. Journal of Applied Biosciences 60: 4410 4420

22. Ngwako S., Balole T.V., Mogotsi K.K., Kwerepe B.C., Sesay A. (2011). Growers, consumers and trader's perceptions of Bambara groundnut (Vigna subterranea (L.) Verdc.) in Botswana. International Journal of Tropical Agriculture and Food Systeme, 5(4): 267-273.

23. Ntundu W.H., Bach I.C., Christiansen J.L., Andersen S.B. (2004). Analysis of genetic diversity in bambara groundnut (Vigna subterranea (L.) Verdc.) landraces using (AFLP) markers. African Journal of Biotechnology, 3 (4): 220-225.

24. Ofori K., Kumaga F.K., Tonyigah A. (2006). Morphological characterization and agronomic evaluation of bambara groundnut (Vigna subterranea (L.) Verdc.) germplasm in Ghana. Plant Genetic Resources Newsletter, 145 : 23-28.

25. Onwubiko N.I.C., Odum O.B., Utazi C.O., Poly-Mbah P.C. (2011). Studies on the adaptation of Bambara Groundnut [Vigna Subterranea (L.) Verdc] in Owerri Southeastern Nigeria. New York Scientific Journal 4(2):60-67. 
26. Ouedraogo M., Jeremy T.O., Jean B.T., Didier B., Clémentine B.D., Gnissa K. (2008). Characterization and evaluation of accessions of Bambara groundnut (Vigna subterranea (L.) Verdcourt) from Burkina Faso. Sciences \& Nature, 5 (2): 191-197.

27. Sévérin B. B., Yao D. (2011). Variabilité morphologique et agronomique des variétés traditionnelles de voandzou (Vigna subterranea L. verdc.) de Côte d'Ivoire. Journal of Applied Biosciences, $41: 2820-2835$

28. Touré Y., Koné M., Silué S., Yatty J. (2013). Prospection, collecte et caractérisation agromorphologique des morphotypes de voandzou de la zone savanicole en Côte d'Ivoire. European Scientific Journal 9 (24): 1857-7881.

29. Zerihun T. (2009). Role of Orphan Crops in Enhancing and Diversifying Food Production in Africa. African Technology Development Forum Journal, Volume 6, Issue 3 and 4, 83 p. 